\title{
Development and validation of a predictive model of failed stepping-down of inhaled corticosteroids in adult asthmatics
}

This article was published in the following Dove Press journal:

Patient Preference and Adherence

18 March 2016

Number of times this article has been viewed

\section{Akira Yamasaki ${ }^{1}$ \\ Katsuyuki Tomita ${ }^{2}$ \\ Kazuhiro Kato ${ }^{3}$ \\ Kouji Fukutani ${ }^{3}$ \\ Hiroyuki Sano 4 \\ Yuji Tohda ${ }^{4}$ \\ Eiji Shimizu'}

'Division of Medical Oncology and Molecular Respirology, Department of Multidisciplinary Internal Medicine, Faculty of Medicine, Tottori University, Tottori, ${ }^{2}$ Department of Respiratory Medicine, Yonago Medical Centre, Tottori, ${ }^{3}$ Department of Respiratory Medicine, Sanin Rosai Hospital, Tottori, ${ }^{4}$ Department of Respiratory Medicine and Allergology, Faculty of Medicine, Kinki University, Osaka, Japan
Correspondence: Akira Yamasaki Division of Medical Oncology and Molecular Respirology, Department of Multidisciplinary Internal Medicine, Tottori University, 36-I Nishi-cho, Yonago 683-8504, Tottori, Japan

Tel +8I 859386537

Fax +8I 859386539

Email yamasaki@grape.med.tottori-u.ac.jp
Background: Asthma guidelines suggest stepping-down of inhaled corticosteroids (ICSs) when asthma is stable.

Objective: To determine outcomes of stepping-down and prediction of outcome after steppingdown of ICSs in controlled adult asthma.

Methods: We performed a retrospective study on 21-81 year-old hospital-based outpatients with asthma in Japan. Protocol for stepping-down of ICSs was performed according to the GINA guideline. Failure/success of stepping-down is judged as occurring exacerbation or not for stepping-down of ICSs. Multiple logistic regression analysis was used to develop a prediction model for failed stepping-down, and then was validated by the leave-one-out cross-validation method. Our model of prediction score was calculated using receiver-operating characteristic area under the curve (AUC) analysis. The Nelson-Aalen curve demonstrated time to failure of stepping-down of ICSs.

Results: A total of 126 patients with asthma attempted the stepping-down of ICSs according to the guideline. Of patients with follow-up, 97 (77.0\%) of stepping-down attempts were successful. Using multivariate logistic regression analysis, comorbidity with rhinitis/rhinosinusitis and phlegm grade were independent predictors of failed stepping-down of ICSs, with odds ratios of 3.8 ( $95 \%$ confidence interval, 1.04-13.3; $P=0.04$ ) and 1.3 (95\% confidence interval, $1.01-11.5 ; P=0.04)$, respectively. These variables were then used to build a prediction score in terms of the prediction of failed steppingdown events. When the two variables were added to form the prediction score, the discriminative power of scores calculated by the prediction model using the AUC was 0.75 (range: 0.62-0.88) for naïve prediction and 0.72 (range: $0.60-0.86$ ) after cross-validation. In the time-to-failure models, cumulative hazard ratio of failed stepping-down have fixed 1 year after stepping-down.

Conclusion: Our results suggest that comorbidity with rhinitis/rhinosinusitis and phlegm grade are imperative to predict failed stepping-down of ICSs in controlled patients with adult asthma. Keywords: asthma, ICSs, stepping-down

\section{Introduction}

As the goal of asthma therapy is to maintain long-term control using the smallest amount of medication possible, current asthma guidelines recommend consideration of stepping-down therapy if asthma remains well controlled for at 3 months to reach a minimum effective dose. ${ }^{1,2}$ To our knowledge, based on the data from a systematic review, asthma exacerbations were statistically no more likely among individuals who reduced inhaled corticosteroids (ICSs) compared to those who maintained their ICS dose. $^{3}$ In addition, it has been reported that stepping-down asthma medication is frequently successful, almost $60 \%-70 \% .^{4,5}$ 
A clear plan of care and follow-up is needed when stepping-down ICSs, because some patients are likely to have recurrent exacerbations. ${ }^{6}$ Some factors of severity, ${ }^{7}$ uncontrolled asthma, airway limitation, ${ }^{8}$ and previous exacerbations in a previous year ${ }^{9}$ were predictors of future risks of exacerbations. However, little is known about which factors are associated with decisions to stepping-down of ICSs in asthma. Few reports demonstrated that predictors of recurrence of asthma symptoms after withdrawal of therapy in mild persistent asthmatics were older age ${ }^{10,11}$ and longer disease duration. ${ }^{10}$

We, therefore, conducted a study to develop and validate a simplified clinical prediction score for estimating the likelihood of failed stepping-down of ICSs in patients with adult asthma who have maintained asthma control for $\geq 3$ months. The scores would aid general practice physicians in identifying individuals who are at likelihood of failed stepping-down of ICSs and should have further investigation and management.

\section{Methods}

\section{Patients}

One hundred and twenty-six eligible patients were recruited from three hospitals in Sanin area in the western side of Japan. The diagnosis of asthma was made on the basis of the Global Initiative for Asthma (GINA) guideline. ${ }^{2}$ Participants were required to be aged 20 years and above, have a diagnosis of persistent mild-to-moderate asthma for at least 3 months, be clinically stable, currently treated with ICSs or combination of ICS/long-acting bronchodilator agonist (LABA), and have features of controlled asthma, which are defined in the following manner: a score on the Asthma Control Questionnaire (ACQ) of $<1.5 .^{12}$ Those patients satisfying any of the following criteria were excluded: a diagnosis of chronic obstructive pulmonary disease, as defined by the Global Initiative for Chronic Obstructive Lung Disease guidelines; ${ }^{13}$ a history of near fatal asthma; oral steroid in the preceding 3 months; aspirin intolerance; flow expiratory volume in 1 second $\left(\mathrm{FEV}_{1}\right) \leq 50 \%$ predicted; pregnancy; recurrent lower respiratory tract infections; and the presence of concomitant respiratory disease such as bronchiectasis. The study was approved by the Yonago Medical Centre Committee on Medical Ethics (24-7), and all participants gave written informed consent. The protocol was registered under UMIN 000011565 in the clinical trial registry.

\section{Data accumulation}

We assessed the following characteristics of patients: age, sex, age of onset of asthma, self-reported smoking (never, former, and current), type of onset symptoms (coughpredominant asthma or wheezing-predominant asthma), need for emergency visits or steroid bursts in the last year, comorbid diseases of rhinitis/rhinosinusitis, IgE value, and IgE specific for aeroantigens.

Asthma severity was characterized using asthma of severity (AOS) score. ${ }^{7,14}$ The AOS score ranges from 0 to 28 , with higher scores indicating more severe asthma.

Rhinitis and rhinosinusitis were diagnosed according to symptom-based definition according to the Allergic Rhinitis and its Impact on Asthma ${ }^{15}$ and European Position Paper of Rhinosinusitis and Nasal Polyps guidelines. ${ }^{16}$

Pulmonary function measurements included prebronhchodilator flow expiratory volume in 1 second $\left(\right.$ pre-FEV $\left._{1}\right)$ and postbronchodilator $\mathrm{FEV}_{1}\left(\right.$ post-FEV $_{1}$ ). All pulmonary function measurements at eligibility were expressed as the percentage predicted for a Japanese population, which was calculated using the formula proposed by the Japanese Respiratory Society. ${ }^{17}$

Adherence is measured as the sum of drug supply days between first and last fill dates divided by length of drug therapy. ${ }^{18}$

Assessment of phlegm grade is based on the Leicester Cough Questionnaire (LCQ), according to the question, "In the last 2 weeks, have you been bothered by sputum (phlegm) production when you cough?" A seven-point Likert scale was used throughout the development of the LCQ ranging from 1 to $7: 1=$ every time, $2=$ most times, $3=$ several times, $4=$ some times, $5=$ occasionally, $6=$ rarely, and $7=$ never.

\section{Protocol of stepping-down}

In all of the eligible patients, asthma symptoms have been well controlled for 3 or more months. According to GINA guideline, ${ }^{2}$ stepping-down treatment occurred upon agreement between patient and each respiratory professional, with full discussion of potential consequences including reappearance of symptoms and increased risk of exacerbations. A strategy for reducing ICSs dose was performed according to the following GINA recommendation listed below:

1) When ICSs had been used alone in medium-to-high doses, a $50 \%$ reduction in dose was attempted.

2) Where control had been achieved at a low dose of ICS alone, treatment was switched to once-daily dosing.

3) When asthma had been controlled with a combination of ICS and LABA, dose reduction of ICSs to $50 \%$ was started while continuing the LABA.

Outcome for this study is failure of stepping-down, judged as occurring exacerbation, which is defined as events where symptoms worsen enough to require a change in treatment. ${ }^{19}$ 


\section{Statistical analysis}

Comparison between groups was done using the $\chi^{2}$-statistics, Mann-Whitney $U$-test, and Student's $t$-test. Nelson-Aalen plot of the cumulative hazard, log-rank test, and logistic regression analysis were conducted with $\mathrm{R}$ ( $\mathrm{R}$ Development Core Team 2015). Results are reported as mean (SD), ranges, or percentages of the appropriate denominator. Significance was accepted at the 5\% level.

Logistic regression analysis was used to determine the relationship between the variables and failed stepping-down. Variables with $P<0.2$ in univariate model were considered significant and were entered into the multivariate model. With an expected failed stepping-down rate of $40 \%$, we required 120 attempts (48 failures) to approximately perform multivariate logistic regression with five variables. In the multivariate model, variables with $P \leq 0.05$ were considered significant. The final model was obtained with multivariate logistic regression and submitted to internal validation with leave-one-out analysis. Model's performances of the final model measured by the goodness-of-fit statistic and the area under the curve (AUC) were assessed. The HosmerLemeshow test is a statistical test for goodness of fit for logistic regression models. A risk score was created using the $\beta$ coefficients of logistic regression model. Individuals were allocated scores relevant to each variable and summed up.

For analysis of time-to-failure, time-to-first occurrence of failure of stepping-down was calculated from start of stepping-down until exacerbations, patients were considered as censored at the end of stepping-down. Risk of occurrence of failure of stepping-down over time was displayed using Kaplan-Meier and Nelson-Aalen curves. ${ }^{20}$

\section{Results}

\section{Patients}

Table 1 lists the general characteristics of patients at the time of starting stepping-down of ICSs. In the eligible 126 patients who were enrolled, all were well controlled as graded on the basis of ACQ $(0.05 \pm 0.15)$. Of the patients

Table I Baseline demographics and clinical characteristics of patients included in analyses

\begin{tabular}{|c|c|c|c|c|}
\hline & Total $(n=\mid 26)$ & $\begin{array}{l}\text { Success of stepping- } \\
\text { down }(n=97)\end{array}$ & $\begin{array}{l}\text { Failure of stepping- } \\
\text { down }(n=29)\end{array}$ & P-value \\
\hline Age, yr & $54.3 \pm 16.3$ & $55.4 \pm 17.1$ & $51.3 \pm 13.9$ & 0.11 \\
\hline Sex, \% female & 73 & 68 & 90 & 0.03 \\
\hline Duration of asthma, yr & $9.6 \pm 13.1$ & $11.7 \pm 14.5$ & $3.7 \pm 3.8$ & 0.07 \\
\hline BMI & $22.4 \pm 3.7$ & $22.5 \pm 3.6$ & $22.3 \pm 4.0$ & 0.78 \\
\hline Cough predominant, \% & 33 & 26 & 55 & 0.02 \\
\hline Rhinitis/rhinosinusitis, \% & 15 & 11 & 30 & 0.002 \\
\hline Family history of asthma & 35 & 31 & 50 & 0.12 \\
\hline \multicolumn{5}{|l|}{ Smoking status } \\
\hline Smoker/ex-smoker/no & $8 / 23 / 95$ & $7 / 20 / 70$ & $1 / 3 / 25$ & 0.46 \\
\hline IgE, IU/L & $367 \pm 972$ & $450 \pm 1,119$ & $|33 \pm| 4 \mid$ & 0.16 \\
\hline $\mathrm{FEV}_{1}$, \%predicted & $94.0 \pm 19.4$ & $92.6 \pm 19.9$ & $97.9 \pm 18.0$ & 0.48 \\
\hline SOA & $5.3 \pm 2.6$ & $5.2 \pm 2.5$ & $5.6 \pm 2.9$ & 0.93 \\
\hline Phlegm grade & $5.8 \pm 1.2$ & $5.9 \pm 1.1$ & $5.4 \pm 1.6$ & 0.07 \\
\hline Adherence & $0.85 \pm 0.24$ & $0.90 \pm 0.16$ & $0.88 \pm 0.19$ & 0.21 \\
\hline Dosage of ICSs ${ }^{a}$ & & & & 0.24 \\
\hline High dose, n (\%) & $22(18)$ & $17(18)$ & $5(20)$ & 0.97 \\
\hline Moderate dose, n (\%) & $67(53)$ & $55(57)$ & $12(40)$ & 0.15 \\
\hline Low dose, n (\%) & $37(28)$ & $25(25)$ & $12(40)$ & 0.11 \\
\hline Type of ICSs & & & & 0.44 \\
\hline Fluticasone, n (\%) & $72(57)$ & $56(58)$ & $16(55)$ & 0.81 \\
\hline Budesonide, n (\%) & $47(37)$ & $37(38)$ & $10(34)$ & 0.72 \\
\hline Beclomethasone HFA, n (\%) & $7(6)$ & $4(4)$ & $3(\mathrm{II})$ & 0.20 \\
\hline Combination therapy, n (\%) & $93(73)$ & $61(63)$ & $22(76)$ & 0.20 \\
\hline Types of LABAs & & & & 0.12 \\
\hline Salmeterol, n (\%) & $57(6 I)$ & $44(72)$ & I 3 (59) & 0.96 \\
\hline Formoterol, n (\%) & $26(39)$ & $17(28)$ & $9(4 I)$ & 0.45 \\
\hline \multicolumn{5}{|l|}{ Other antiasthmatics } \\
\hline Antileukotrienes, n (\%) & II (9) & $7(7)$ & $4(14)$ & 0.28 \\
\hline Nasal steroids, n (\%) & $9(8)$ & $5(5)$ & $4(14)$ & 0.21 \\
\hline
\end{tabular}

Notes: Unless otherwise stated values are mean \pm SD. aICS dose and dose equivalence were classified according to the BTS guidelines ${ }^{30}$ as I) high dose $(\geq 800 \mu g / d$ beclomethasone (BDP) equivalent); 2 ) moderate dose (400-800 $\mu \mathrm{g} / \mathrm{d}$ BDP); 3 ) low dose ( $<400 \mu \mathrm{g} / \mathrm{d}$ BDP) and dose equivalence for BDP:budesonide at a I:I ratio and BDP:fluticasone at a 2:I ratio.

Abbreviations: BMI, body mass index; FEV , forced expiratory volume in I second; HFA, hydrofluoroalkane; ICSs, inhaled corticosteroids; LABAs, long-acting bronchodilator agonists; SOA, severity of asthma; yr, years. 
with follow-up, 29 (23.0\%) were identified from failed stepping-down attempts. Of the 29 failed stepping-down attempts, all were judged to need to return to the previous ICS dose due to loss of asthma control. Two needed steroid bursts, but none required hospitalization and emergency care. There was no significant difference in the serum housedust-mite-specific IgE levels between the two groups. When adherence was measured as the sum of drug supply days between the first and last fill dates divided by the length of drug therapy, there was no significant difference between the two groups.

\section{Predictors for failed stepping-down}

All variables with $P$-values $<0.2$ were simultaneously included in a multivariate model, as listed in Table 2. The final model contained only two variables (ie, comorbidity with rhinitis/rhinosinusitis and phlegm grade), and their odds ratio (ORs) are listed in Table 2. The scoring scheme for each variable was constructed by rounding off the ORs and assigning 1 to the reference category. A total score of 2 demonstrated the score discriminative ability as 0.77 of AUC. The goodness of fit was assessed, and it was found that the final model was fit well with our data (the Hosmer-Lemeshow test, $P=0.264$ ).

When the two variables were added to form the prediction score, the AUC was 0.75 (range: 0.62-0.88) for naïve prediction and 0.72 (range: 0.60-0.86) after cross-validation. The $C$ statistics of the derivative and validated models were very much similar, ie, 0.75 and 0.72 . The average difference (known as degree of optimism) was 0.03 . Hence, this analysis allows to rule out substantial overfitting of the prediction score.

When the scores for failure rate were compared, failure of stepping-down attempts occurred in 4 of 44 (9\%) patients with "Score 0", 9/51 (18\%) with "Score 1", and 16/31 (52\%) with "Score 2".

For analysis of time to failure, the average of duration of follow-up was 13 months (7-1,037 days); $108 \pm 90$ days
(7-379 days) for the failed patients; and 413 \pm 220 days (78-1,037 days) for the patients who ultimately succeeded in their stepping-down attempts. Among each score, total score as 2 is shown as higher risk of failed stepping-down in Kaplan-Meier curve (Figure 1A). The risk of failed stepping-down also can be interpreted in terms of cumulative hazard curve, which graphically displays the risk experienced over the entire follow-up period. As shown in Figure 1B, visual inspection of the Nelson-Aalen curve reveals that cumulative hazard ratio of failed stepping-down has been fixed at 1 year after stepping-down.

\section{Discussion}

We have developed and validated a simplified clinical prediction score for failed stepping-down of ICSs in patients with controlled asthma. The scoring was made easier and more simplified as it used factors that were readily available and were simple to assess even in clinical practice.

In this study, approximately $70 \%$ of patients with controlled asthma could step-down ICSs according to the current guidelines. Our results are in accordance with Leuppi's report that determined that the majority of patients could undergo a halving of their ICS dose without exacerbation. ${ }^{11}$ We mainly focus on estimating likelihood of failed stepping-down of ICSs. In our study, the predictors of failed stepping-down were comorbidity with rhinitis/rhinosinusitis and degree of sputum. In addition, we have validated the clinical prediction score using a leave-one-out technique, which is considered a good technique for internal validation. ${ }^{21}$ The AUC of the simplified score was fair in both derivative and leave-one-out validated data (ie, 0.75 and 0.72 ), indicating that the score can discriminate well between individuals who were successful and those who failed. Our model is simplified and should be easy to apply in clinical practice because the required variables are routinely measured.

Our results that comorbidity with rhinitis/rhinosinusitis and phlegm grade might contribute to be failure of stepping-down of ICSs have been predictable, because some

Table 2 Factors associated with successful stepping-down of ICSs and creating a scoring scheme: multiple logistic regression analysis

\begin{tabular}{|c|c|c|c|c|c|}
\hline Variables & Coefficient & SE & OR $(95 \% \mathrm{CI})$ & $P$-value & $\begin{array}{l}\text { Scoring score } \\
\text { for individual }\end{array}$ \\
\hline Sex: female & -0.76 & 0.90 & $0.47(0.08-2.72)$ & 0.40 & 0 \\
\hline Duration of asthma: 7 years or more & -0.12 & 0.68 & $0.89(0.23-3.35)$ & 0.86 & 0 \\
\hline Type: cough predominant & -0.59 & 0.66 & $0.55(0.15-2.01)$ & 0.37 & 0 \\
\hline Family history of asthma: yes & 0.68 & 0.65 & $1.97(0.55-7.09)$ & 0.30 & 0 \\
\hline Phlegm grade: 5 or less (occasionally) & -1.13 & 8.50 & $0.32(0.09-0.98)$ & 0.04 & I \\
\hline Comorbidity: rhinitis/rhinosinusitis & -1.30 & 3.81 & $1.27(0.07-0.97)$ & 0.04 & I \\
\hline
\end{tabular}

Abbreviations: $\mathrm{Cl}$, confidence interval; ICSs, inhaled corticosteroids; OR, odds ratio; SE, standard error. 
A

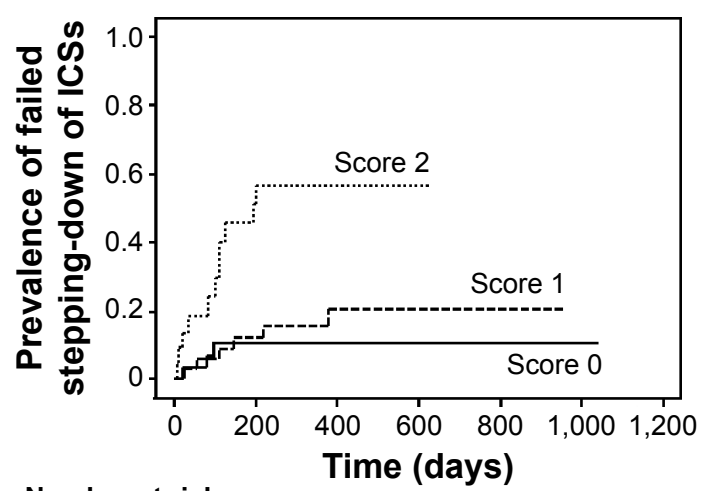

Number at risk

$\begin{array}{lllllll}\text { Score 0 } & 44 & 31 & 21 & 9 & 3 & 1 \\ \text { Score 1 } & 51 & 40 & 23 & 7 & 4 & 0 \\ \text { Score 2 } & 31 & 21 & 8 & 3 & 0 & 0\end{array}$

B

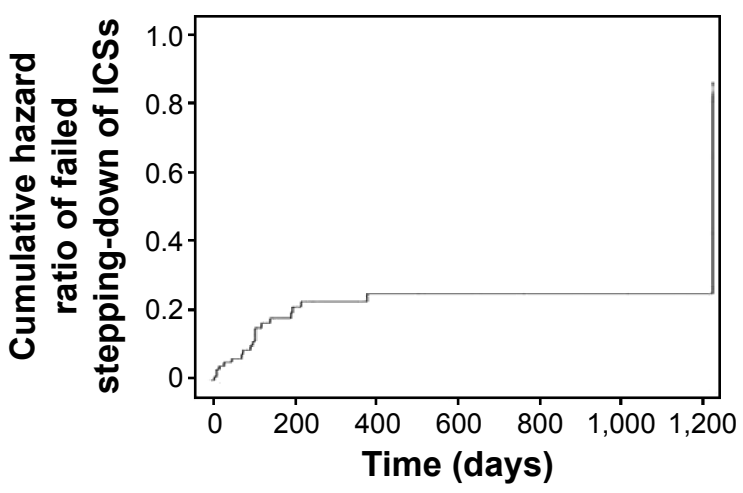

Figure I Time to treatment failure during stepping-down of ICSs.

Notes: The cumulative percentage and hazard ratio of patients with failed stepping-down of ICSs was estimated by using the Kaplan-Meier method (A) and Nelson-Aalen plot (B). In cumulative percentage of failed stepping-down of ICSs using Kaplan-Meier method, solid line - score 0 , hatched line - score I, and broken line - score 2 are displayed in prediction score for failed stepping-down of ICSs.

Abbreviation: ICSs, inhaled corticosteroids.

reports have shown that these variables were useful tools for predicting exacerbation. It was reported that patients with rhinitis/rhinosinusitis had increased asthma exacerbations. ${ }^{22}$ In addition, chronic mucus hypersecretion that is characterized with chronic cough and sputum production occurs frequently in adults with stable asthma and is associated with more exacerbations in never smokers. ${ }^{23}$ The reason that severity of asthma and lung function was not predictor of stepping-down failure might be explained by degree of severity. Our eligible patients with asthma were totally controlled and had low severity score of asthma.

Biomarkers evaluated for controlled condition of asthma included methacholine airway hyperresponsiveness, ${ }^{24}$ sputum eosinophils, ${ }^{25}$ and exhaled nitric oxide. ${ }^{26,27}$ While these approaches have shown promise in reducing exacerbations and/or improving asthma control, although performed in many subspecialty practices and research centers, they are not routinely available in most primary care practices.

Our results of treatment-to-failure analysis demonstrated that no exacerbations with halving ICSs for 1 year might suggest stable conditions of asthma. ICS treatment may be stopped if the patient remains well controlled on the lowest dose of ICS for 1 year according to GINA guideline.

There are some limitations in this report. First, an intrinsic limitation of our study design is that we could not confirm that patients were on the minimum does that maintained asthma control before study start. Thus, the overtreatment quite commonly reported in real-life conditions ${ }^{28,29}$ cannot be excluded. However, patients were selected from pulmonary centers where they were regularly assessed and treated according to the current guidelines, suggesting that the dose of the original ICS/LABA prescription was appropriate. The second limitation is the sample size. A larger sample size would have allowed more power to analyze the subgroup of responders that halve ICSs; therefore, we cannot exclude that lung function may be an important factor in decisions to stepdown ICSs. Despite these limitations, we have preliminary evidence that having our prediction score may be important for patients and providers who are deciding on stepping-down of ICSs. As this study was performed retrospectively, future work will be necessary using a prospective design including external validation.

Our results demonstrated that reducing the dose of ICSs by $50 \%$ appears to be the safest method of stepping-down in accordance with the guidelines. We propose a simple clinical prediction score of failed stepping-down of ICSs.

\section{Disclosure}

The authors report no conflicts of interest in this work.

\section{References}

1. National Asthma Education and Prevention Program. Expert Panel Report 3 (EPR-3) 2007: Guidelines for the Diagnosis and Management of Asthma. Bethesda, MD: Department of Health and Human Services, National Institutes of Health, National Heart, Lung, and Blood Institute, National Asthma Education and Prevention Program; 2007. NIH Publication Number 08-5846.

2. Global Strategy for Asthma Management and Prevention, Global Initiative for Asthma (GINA) 2014. Available from: http://www.ginasthma.org/. Accessed January 11, 2016. 
3. Hagan JB, Samant SA, Volcheck GW, et al. The risk of asthma exacerbation after reducing inhaled corticosteroids: a systematic review and meta-analysis of randomized controlled trials. Allergy. 2014;69:510-516

4. Rank MA, Branda ME, McWilliams DB, et al. Outcomes of stepping down asthma medications in a guideline-based pediatric asthma management program. Ann Allergy Asthma Immunol. 2013;110: 354-358.e2

5. Tsurikisawa N, Oshikata C, Tsuburai T, et al. Markers for step-down of inhaled corticosteroid therapy in adult asthmatics. Allergol Int. 2012; 61:419-429.

6. Rank MA, Peters SP. The risks, benefits, and uncertainties of stepping down asthma medications. J Allergy Clin Immunol Pract. 2014;2:503-509.

7. Eisner MD, Yegin, A, Trzaskoma B. Severity of asthma score predicts clinical outcomes in patients with moderate to severe persistent asthma. Chest. 2012;141:58-65.

8. Sato R, Tomita T, Sano H, et al. The strategy for predicting future exacerbation of asthma using a combination of the asthma control test and lung function test. J Asthma. 2009;46:677-682.

9. Tomita K, Sano H, Iwanaga T, et al. Association between episodes of upper respiratory infection and exacerbations in adult patients with asthma. J Asthma. 2012;49:253-259.

10. Tonelli M, Bacci E, Dente FL, et al. Predictors of symptom recurrence after low-dose inhaled corticosteroid cessation in mild persistent asthma. Respir Med. 2006;100:622-629.

11. Leuppi JD, Salome CM, Jenkins CR, et al. Predictive markers of asthma exacerbation during stepwise dose reduction of inhaled corticosteroids. Am J Respir Crit Care Med. 2001;163:406-412.

12. Juniper EF, Bousquet J, Abetz L, Bateman ED. Identifying "wellcontrolled" and "not well-controlled" asthma using the Asthma Control Questionnaire. Respir Med. 2006;100:616-621.

13. GOLD Report Executive Summary. Global Strategy for Diagnosis, Management, and Prevention of COPD. December 2009. GOLD-Global Initiative for Chronic Obstructive Lung Disease home page. Available from: http://www.goldcopd.org. Accessed January 11, 2016.

14. Eisner MD, Katz PP, Yelin EH, et al. Assessment of asthma severity in adults with asthma treated by family practitioners, allergists, and pulmonologists. Med Care. 1998;36:1567-1577.

15. Bousquet J, Khaltaev N, Cruz AA, et al. Allergic Rhinitis and its Impact on Asthma (ARIA) 2008 update (in collaboration with the World Health Organization, GA(2)LEN and AllerGen). Allergy. 2008;63:8-160.

16. Thomas M, Yawn BP, Price D, Lund V, Mullol J, Fokkens W. EPOS primary care guidelines: European position paper on the primary care diagnosis and management of rhinosinusitis and nasal polyps 2. Prim Care Respir J. 2008;17:79-89.

17. The Committee of Respiratory Physiology on Japan Society of Thoracic Disease. Reference values for spirometry, including vital capacity, in Japanese adults calculated with the LMS method and compared with previous values. Respir Investig. 2014;52:242-250.
18. Bender B, Milgrom H, Rand C. Nonadherence in asthmatic patients: is there a solution to the problem? Ann Allergy Asthma Immunol. 1997;79: 177-185.

19. Reddel HK, Taylor DR, Bateman ED, et al; on behalf of the American Thoracic Society/European Respiratory Society Task Force on Asthma Control and Exacerbations. An official American Thoracic Society/ European Respiratory Society Statement: asthma control and exacerbations. Am J Respir Crit Care Med. 2009;180:59-99.

20. Chongsuvivatwong V. Survival analysis. In: McNeil EB, editor. Analysis of Epidemiological Data Using $R$ and Epicalc. 2. Songkhla: Chanmuang Press; 2008:225-236.

21. Bautista D, Arana E, Marti-Bonmati L, Paredes R. Validation of logistic regression models in small samples: application to calvarial lesions diagnosis. J Clin Epidemiol. 1999;52:237-241.

22. Dixon AE, Kaminsky DA, Holbrook JT, Wise RA, Shade DM, Irvin CG. Allergic rhinitis and sinusitis in asthma: differential effects on symptoms and pulmonary function. Chest. 2006;130:429-435.

23. Thomson NC, Chaudhuri R, Messow CM, et al. Chronic cough and sputum production are associated with worse clinical outcomes in stable asthma. Respir Med. 2013;107:1501-1508.

24. Sont JK, Eillems LN, Bel EH, et al. Clinical control and histopathologic outcome of asthma when using airway hyperresponsiveness as an additional guide to long-term treatment. The AMPUL Study Group. Am J Respir Crit Care Med. 1999;159:1043-1051.

25. Green RH, Brightling CE, McKenna S, et al. Asthma exacerbations and sputum eosinophil counts: a randomized controlled trial. Lancet. 2002;360:1715-1721.

26. Smith AD, Cowan JO, Brassett KP, Herbison GP, Taylor DR. Use of exhaled nitric oxide measurements to guide treatment in chronic asthma. N Engl J Med. 2005;352:2163-2173.

27. Szefler SJ, Mitchell H, Sorkness CA, et al. Management of asthma based on exhaled nitric oxide in addition to guideline-based treatment for inner-city adolescents and young adults: arandmised controlled trial. Lancet. 2008;372:1065-1072.

28. Diette GB, Patino CM, Merriman B, et al. Patient factors that physicians use to assign asthma treatment. Arch Intern Med. 2007;167: 1360-1366.

29. Manoharan A, Lipworth BJ, Craig E, Jackson C. The potential role of direct and indirect bronchial challenge testing to identify overtreatment of community managed asthma. Clin Exp Allergy. 2014;44: 1240-1245.

30. British Thoracic Society/Scottish Intercollegiate Guidelines Network. Guideline on the management of asthma. Thorax. 2003;58(Suppl I): $1-192$.
Patient Preference and Adherence

\section{Publish your work in this journal}

Patient Preference and Adherence is an international, peer-reviewed, open access journal that focuses on the growing importance of patient preference and adherence throughout the therapeutic continuum. Patient satisfaction, acceptability, quality of life, compliance, persistence and their role in developing new therapeutic modalities and compounds to optimize
Dovepress

clinical outcomes for existing disease states are major areas of interest for the journal. This journal has been accepted for indexing on PubMed Central The manuscript management system is completely online and includes a very quick and fair peer-review system, which is all easy to use. Visit http://www. dovepress.com/testimonials.php to read real quotes from published authors. 